\title{
Modular Transmitter and Receiver for Space-Time Coding
}

\author{
Quinten Van den Brande ${ }^{1}$, Patrick Van Torre ${ }^{2}$, Jo Verhaevert ${ }^{3}$, Jan Vanfleteren ${ }^{4}$, Hendrik Rogier ${ }^{5}$ \\ $1,2,3,5$ Department of Information Technology, Ghent University/iMinds, \\ Quinten.VandenBrande@UGent.Be, Patrick.VanTorre@UGent.Be, Jo.Verhaevert@UGent.Be, Hendrik.Rogier@UGent.Be \\ ${ }^{4}$ Department of Electronics and Information Systems, Ghent University/CMST, Jan.Vanfleteren@UGent.Be, \\ Technologiepark-Zwijnaarde 15, 9052 Ghent, Belgium
}

\begin{abstract}
In this article a Multiple Input Single Output (MISO) hardware design is reported, comprising both a transmitter and a receiver for space-time coded signals. The transmitter implements Alamouti space-time coding, while the receiver uses maximum likelihood estimation to decode the space-time codes. As a result, the hardware communication system is configured as a $2 \times 1$ MISO link. The hardware is designed modularly, in order for the system to be easily extendible for the connection of multiple antennas. A digital core allows high-speed data generation in the signal generator units, forwarding analog $I$ and $Q$ signals to the radio-frequency modules connected to it. The Bit Error Rate (BER) performance of the Alamouti diversity scheme is compared to the performance of Single Input Single Output (SISO) and Maximum Ratio Combining (MRC) systems. This comparison is based on simulation results, providing a good approximation of the theoretical BER performance, and a series of hardware measurements to empirically support this theoretical performance.
\end{abstract}

Index Terms-RF circuit design, Space-time block coding, Alamouti, diversity, Bit Error Rate, Multiple Input Multiple Output.

\section{INTRODUCTION}

The trade-off between reliability and data rate is a wellknown problem in wireless transceiver systems. The Shannon channel capacity theorem [1] specifies a theoretical upper limit for the channel capacity of such a system, which in turn limits the maximum reliability and data rate. However, this limitation can be overcome with the use of Multiple Input Multiple Output (MIMO) systems. The use of MIMO transceiver systems introduces a new trade-off between diversity and spatial multiplexing gain, thus presenting the designer a choice between transmission reliability and data rate respectively, or a balanced mix of both.

Diversity techniques come in many forms and are used to mitigate the effects of fading over a communication link. However, most implementations of such techniques are relegated to the receiver end, resulting in a higher power consumption and a decreasing battery life in portable devices. Spacetime coding is a transmit diversity technique that maximizes diversity gain by sending multiple versions of the transmitted symbols across a number of antennas, thus providing the receiver with these various symbol versions to improve the reliability of the data transfer, as described in [2]. This way, a diversity gain comparable to receiver diversity gain can be achieved by using multiple transmit antennas at wireless base stations, hence preventing excess power consumption on portable devices, as described in [3]. Many forms of spacetime coding schemes exist, for different MIMO configurations. In order to pursue further research, a low-cost, high performance modular hardware system is necessary to support this variety of space-time codes. This article describes the architecture and performance of the designed hardware, with an implementation of the Alamouti space-time coding scheme in a 2x1 MISO configuration as an example.

In Section II, the Alamouti coding scheme is presented. It is followed in Section III by an overview of the hardware that was built to allow a practical low-cost realization. The Alamouti coding scheme is verified by a simulation model, providing an image of its BER characteristics in Section IV. Finally, the results of a hardware measurement will be provided in Section V, in order to demonstrate the functionality of the system. Section VI outlines the conclusions.

\section{SPACE-TIME CODING: Alamouti DiVERSity SCHEME}

In [4] a space-time coding diversity scheme is proposed for 2x1 MISO and 2x2 MIMO systems, widely known as the Alamouti diversity scheme. To implement this scheme for a 2x1 MIMO system, three major components are needed: the Alamouti encoder, an MRC and a Maximum Likelihood Estimator (MLE), all described below.

The Alamouti encoding scheme takes subsequent constellation symbols in pairs and combines them into a space-time block code. As seen in equation 1, this block is then sent from two transmit antennas in two subsequent time slots.

$$
C=\left(\begin{array}{cc}
s_{0} & s_{1} \\
-s_{1}^{*} & s_{0}^{*}
\end{array}\right)
$$

In time slot $1 s_{0}$ and $s_{1}$ are sent from transmit antenna 1 and 2 respectively. In the second time slot $-s_{1}^{*}$ and $s_{0}^{*} *$, where $*$ denotes the complex conjugate, are sent in the same configuration.

On the receiver side, the transmitted signals from transmit antenna 1 and 2 are captured onto two receive antennas. The received signals at both time slots can be described as

$$
\begin{aligned}
& r_{0}=h_{0} s_{0}+h_{1} s_{1}+n_{0} \\
& r_{1}=-h_{0} s_{1}^{*}+h_{1} s_{0}^{*}+n_{1},
\end{aligned}
$$


where $h_{i}$ represents the complex channel coefficient of the channel between the receive antenna and transmit antenna $i$. The added noise at time slot $i$ is represented by $n_{i}$.

Assuming the receiver knows both channel coefficients, the MRC combines the received signals as

$$
\begin{aligned}
& \tilde{s}_{0}=h_{0}^{*} r_{0}+h_{1} r_{1}^{*} \\
& \tilde{s}_{1}=h_{1}^{*} r_{0}-h_{0} r_{1}^{*} .
\end{aligned}
$$

The combined signals $\tilde{s}_{0}$ and $\tilde{s}_{1}$ are then sent to the MLE unit to determine for each signal $s_{0}$ and $s_{1}$, which constellation symbol was most likely transmitted. Here Quadrature Phase Shift Keying (QPSK) was used to transmit data, hence the decision rule in [4] may be simplified to

choose $s_{i}$ if

$$
d^{2}\left(\tilde{s}_{0}, s_{i}\right) \leq d^{2}\left(\tilde{s}_{0}, s_{k}\right) \quad \forall i \neq k .
$$

The MLE then produces the signals $\hat{s}_{0}$ and $\hat{s}_{1}$, for which (6) is true.

\section{HARDWARE}

In order to empirically support the premises made in [4] and [5] concerning the BER performance of space-time coding, a hardware communication system prototype was developed to support the use of multiple space-time coding techniques in a variety of MISO configurations.

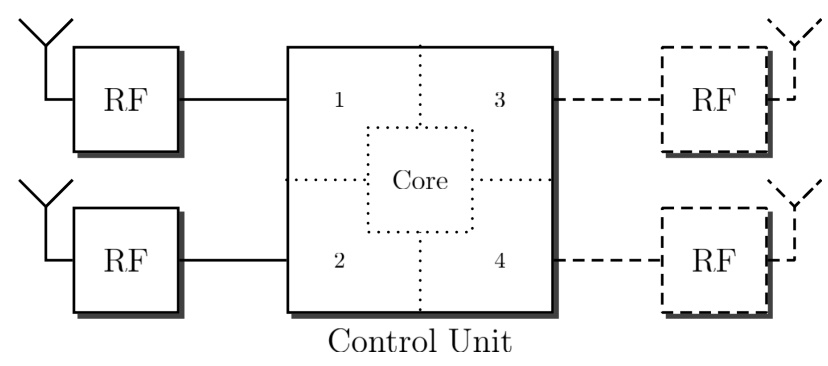

Fig. 1. Modular design of transmitter (maximum of 4 RF modules).

The requirement of a multifunctional hardware system desires a modular design. The transmitter is hence composed of one central control unit and multiple RF modules, as shown in Figure 1. As a result, different MISO configurations can be implemented simply by adding or removing RF modules from the control unit. The control unit is divided into one central core, responsible for the communication between software and hardware, and four signal generator units, each one dedicated to one specific RF module. Each signal generator unit consists of a 16-bit Static Random Access Memory (SRAM) and two 8bit Digital-to-Analog Converters (DAC), enabling synchronous transmission on all RF modules by reading out the SRAM content with a synchronous clock signal. The control unit PCB is displayed on Figure 2, with the signal generator units and central core on the top and bottom layer respectively. The receiver side is implemented using a DVB-T USB dongle, which captures the wireless signal and forwards it to MATLAB for post-processing [6].

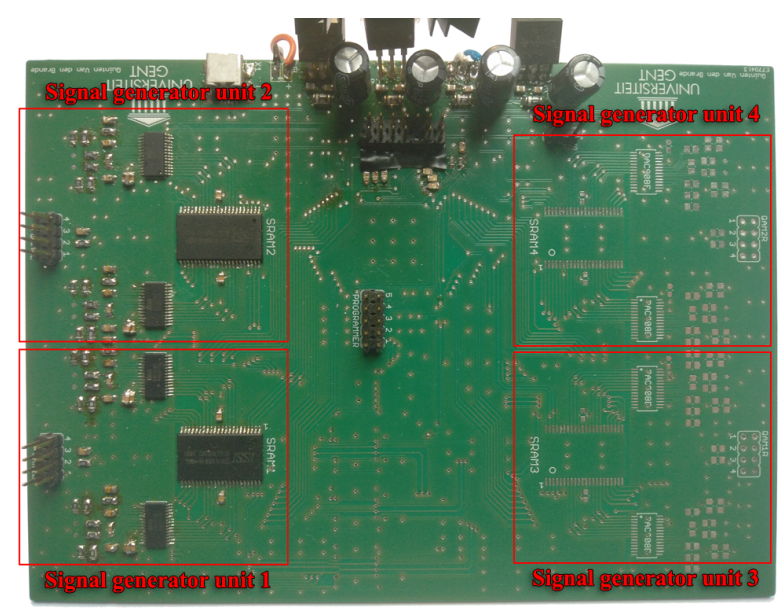

Fig. 2. Control unit PCB with signal generator units and central core on top and bottom layer respectively.

To implement Alamouti space-time block coding, a software model is developed in MATLAB [7]. This software consists of a PSK (Phase Shift Keying) modulator, which modulates a known pseudo-random bit stream to QPSK or BPSK (Binary PSK) symbols, and a space-time encoder. Once the bit stream is encoded, it is sent to the control unit where the central core assigns every symbol in the space-time block code to the correct signal generator unit by storing it in the corresponding SRAM. When data storage is complete, the central core starts a synchronous clock which activates all signal generator units, thus iterating the SRAM content and passing them on to the corresponding RF module where the data is transmitted. As an example, the full block diagram of the transmitter, implementing Alamouti space-time coding, is represented in Figure 3. In order to decode the space-time coding the total communication system may be configured as a 2x1 MISO or 2x2 MIMO system, each of them implementing a different decoding scheme.

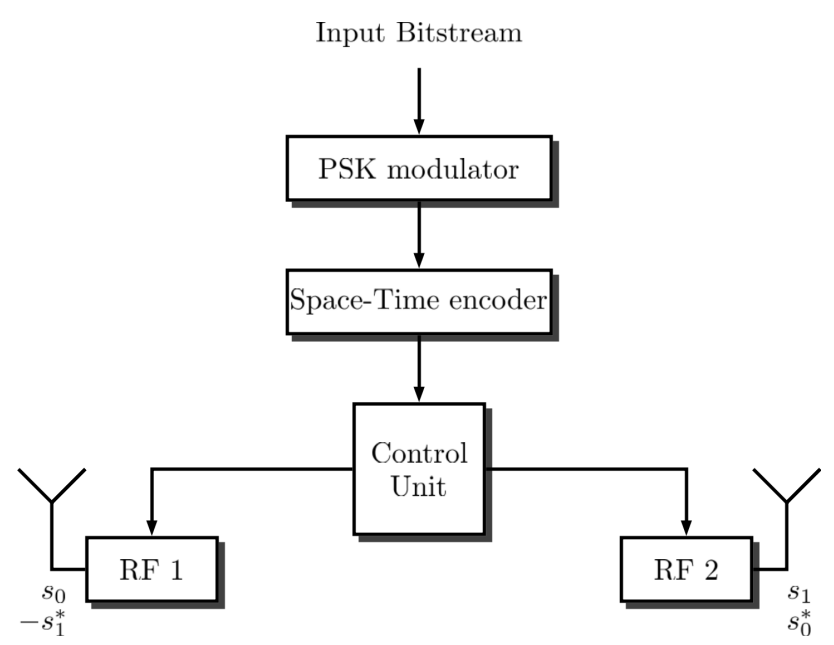

Fig. 3. Transmitter block diagram implementing Alamouti space-time block coding. 
On the receiver side the Alamouti space-time encoded wireless data is captured using a DVB-T dongle [8], thereby implementing a $2 \times 1$ MISO communication system. The DVB-T dongle is a USB stick containing an RF tuner, converting to a zero-IF (Intermediate Frequency), followed by another chip to sample the IQ-values (In-phase and Quadrature). The IQ-samples are sent to MATLAB, where it is decoded and demodulated for BER calculations. Our MATLAB SDR (Software Defined Radio) consists of a channel estimator, an MRC, an MLE and a PSK demodulator. In order to estimate the channel parameters each transmit antenna sends a sequence of known pilot symbols in separate time slots. Subsequently, the channel estimator compares each received pilot sequence with the corresponding known pilot sequence, resulting in an estimation of the channel parameters $h_{0}$ and $h_{1}$ for a $2 \times 1$ MISO system. The MRC uses these parameters to construct the combined signals $\tilde{s}_{0}$ and $\tilde{s}_{1}$, which are fed to the MLE to produce the maximum likelihood estimates $\hat{s}_{0}$ and $\hat{s}_{1}$. Finally, these symbols are sent to the PSK demodulator, which in turn converts the estimated PSK symbols into the received bit stream. The BER can be calculated by comparing the input and output bit stream. The complete block diagram of the receiver is shown in Figure 4.

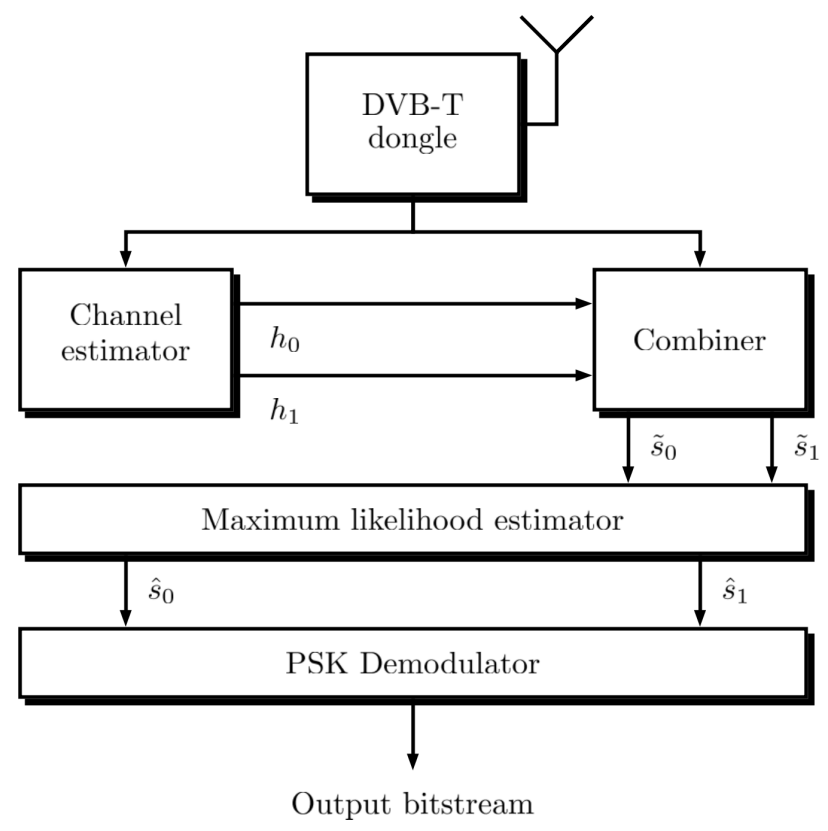

Fig. 4. Receiver block diagram for a 2x1 MISO Alamouti space-time block code system.

\section{BIT ERROR RATE}

The use of space-time coding schemes provides diversity gain, leading to a lower BER for a given $E_{b} / N_{0}$ (Energy per Bit to Spectral Noise Density ratio) in Rayleigh fading channels. The relation between $E_{b} / N_{0}$ and BER varies for different MISO configurations but, in general, the use of more transmit antennas results in a lower BER. To verify the BER performance of Alamouti space-time coding and to create a reference point for hardware measurements, a simulation model is developed in MATLAB. This model implements both the transmitter and receiver side, specified in Section III, as well as a channel emulator for adding Additive White Gaussian Noise (AWGN) and Rayleigh fading to the transmitted signal.

\section{A. Simulation results}

In order to get a good representation of the theoretical BER performance, the simulation is performed for 1 million BPSK symbols for each Energy per Bit to Spectral Noise Density ratio $\left(E_{b} / N_{0}\right)$ in the range $[-5 ; 20] \mathrm{dB}$. Note that at every new iteration, the channel emulator is reinitialized to ensure random added AWGN and Rayleigh fading. Figure 5 shows the BER performance of a $2 \times 1$ MISO Alamouti system as a function of $E_{b} / N_{0}$ in $\mathrm{dB}$, with

$$
E_{b} / N_{0}=\frac{\mathrm{SNR}}{n},
$$

where SNR is the Signal-to-Noise Ratio and $n$ the number of bits per symbol. As a point of reference, the BER performance of a SISO and 1x2 Single Input Multiple Output (SIMO) with MRC system in Rayleigh fading conditions is added to the simulation.

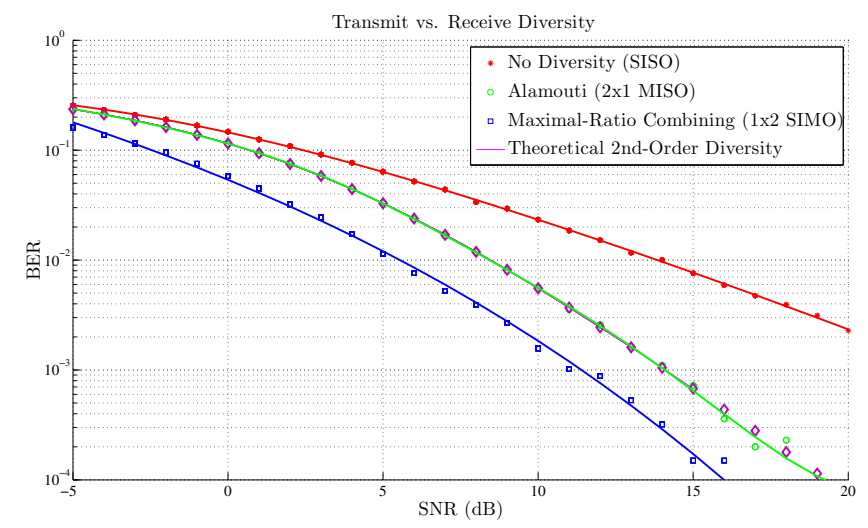

Fig. 5. Simulation result log-scaled BER performance as a function of $E_{b} / N_{0}$ [dB].

Figure 5 shows that the BER performance is greatly enhanced by the use Alamouti space-time coding in a $2 \times 1$ MISO configuration, in comparison to the SISO configuration. Although the MRC SIMO system has an improved BER performance over Alamouti space-time coding for the same $E_{b} / N_{0}$, both BER curves have a similar slope for the higher $E_{b} / N_{0}$ values in the plot. The $3 \mathrm{~dB}$ difference between both curves can be explained by the fact that for the $1 \times 2$ MRC SIMO system, array gain is also present, where this is not the case for the $2 \times 1$ MISO link. This observation leads to the conclusion that an Alamouti space-time coding system yields a similar diversity gain as an MRC 1x2 SIMO system, thus confirming the premises made in [4] concerning the comparable diversity gain between both systems. 


\section{HARDWARE MEASUREMENTS}

In order to provide empirical support for the theoretical BER performance of space-time coding, a large number of measurements is needed to obtain a high BER resolution. As a result, the functionality of the developed hardware system should first be verified to ensure future channel measurements reflect the true BER performance of the space-time coding. This section describes this verification process.

\section{A. Measurement setup}

The measurement setup, shown in Figure 6, is specified to highlight the possibility of noise or interference on the received signal. Since the equipment used in these measurements can introduce spurious components on the received signal, they should be taken into account.

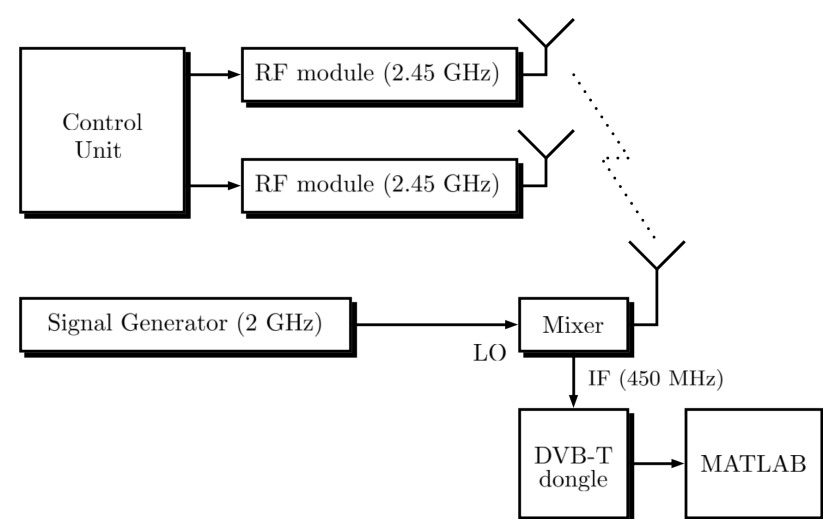

Fig. 6. Block diagram of hardware measuring setup.

To verify the functionality of the hardware system, an Alamouti 2x1 MISO system is implemented. The transmitter, described in Section III, starts its transmission when the data transfer from MATLAB to the control unit is complete. At this point, the RF modules start transmitting at $2.45 \mathrm{GHz}$ simultaneously. On the receiver side, this data is captured by the DVB-T dongle. However, this receiver has a frequency range limited to a maximum frequency of $1.70 \mathrm{GHz}$, making the down-conversion of the RF signal to an intermediate frequency necessary. Therefore a mixer is used, which captures the RF signal and converts it from $2.45 \mathrm{GHz}$ down to $450 \mathrm{MHz}$. Subsequently, this mixed signal is converted to baseband I and $\mathrm{Q}$ by the DVB-T dongle and sent to MATLAB for postprocessing.

\section{B. Measuring results}

In order to verify the correct implementation of Alamouti space-time coding on the hardware system, the received signal is decoded using the MRC and MLE specified in Section II. If the received signals can be decoded using equations (4), (5) and (6) with minimal errors, the designed communication system properly implements the Alamouti coding scheme and thus can be used for further measurements.

Figure 7 shows the amplitude of the received data frame as a function of time, indicating the time slots for the pilot sequences, the space-time encoded data and the gap. Using (7), the $E_{b} / N_{0}$ in $\mathrm{dB}$ of this signal is found to be $10.25 \mathrm{~dB}$.

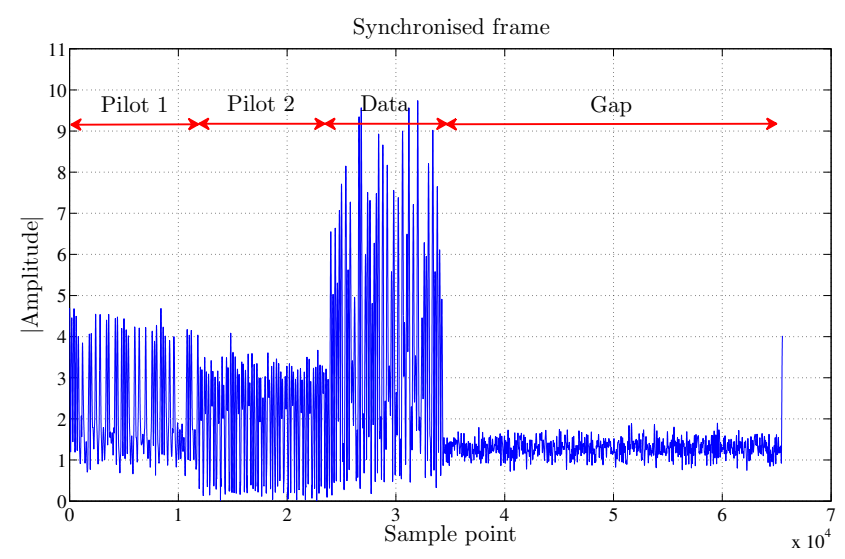

Fig. 7. Amplitude of received data frame.

Next, the channel parameters are estimated by analyzing the received pilot symbols and comparing them to the expected pilot sequence, thus allowing the receiver to extract knowledge about the channel coefficients. As seen in Figure 8, the received pilot symbols have a phase offset with respect to the in-phase axis of $-36.4^{\circ}$ and $36.4^{\circ}$ for pilot sequence 1 and 2 respectively. Using these phase offsets $\Delta \phi_{i}$, as well as the estimated path loss for each antenna $\alpha_{i}$, the complex channel coefficient from transmit antenna $i$ to the receive antenna can be estimated as $h_{i}=\alpha_{i} e^{j \Delta \phi_{i} \frac{2 \pi}{360^{\circ}}}$.

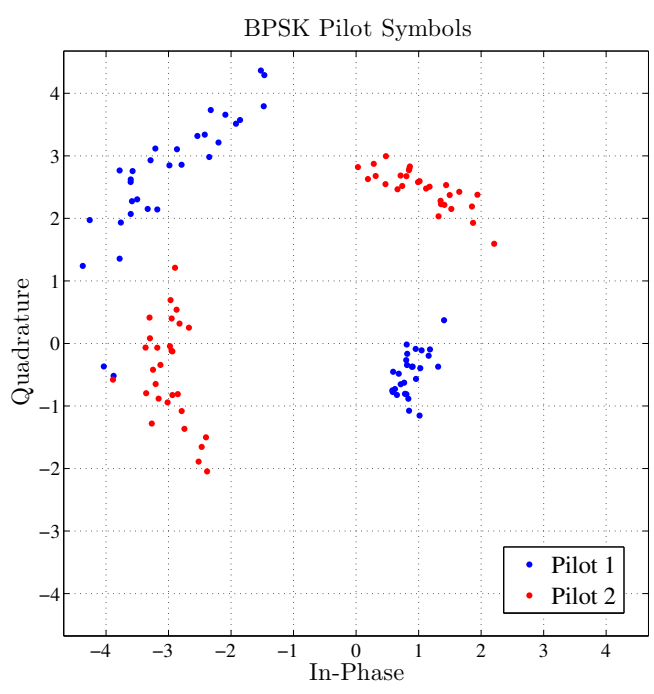

Fig. 8. Received pilot symbols from sequence 1 (blue) and 2 (red).

Once the channel has been estimated, the received spacetime codes can be decoded employing the MRC and MLE. The received symbols are a superposition of QPSK symbols sent from the transmit antennas with their amplitudes and phases modified by the different complex channel coefficients. The MRC combines the received symbols, as specified in Section II, so that the MLE can produce the maximum likelihood 


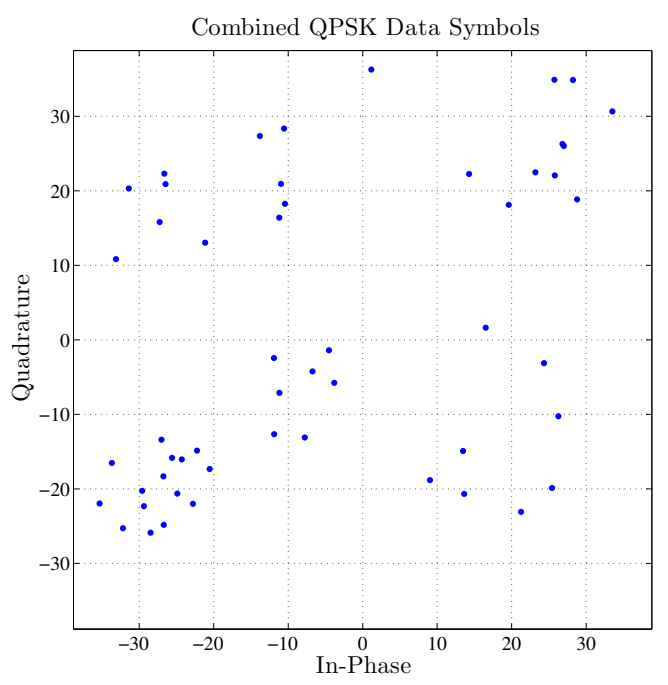

Fig. 9. Combined Alamouti space-time code symbols.

estimates of the received symbols. The combined symbols can be seen in Figure 9, showing clear QPSK constellation clusters with some minor deviations. After MLE, the maximum likelihood estimates are demodulated, creating the received bit stream. Comparing the received bit stream with the input bit stream produces the BER for this transmission, in this case resulting in zero bit errors.

The BER obtained after space-time decoding illustrates the correct functionality of the designed hardware system. However, due to the large phase noise caused by various Phase Locked Loops (PLL) in the measuring setup, a lot of noise is visible in the scatter plot. In addition, when looking at the phase noise of the received pilot symbols in Figure 8, one can see that the accuracy of the channel estimation is limited. A direct comparison to the BER characteristics for Rayleigh fading environments requires a large number of measurements with a better phase noise performance.

Taking this into account, in order to proceed to the empirical verification of the BER performance of space-time coding, the hardware system should first be further optimized for reduced phase noise so that channel estimation can occur with acceptable accuracy. Only after this optimization process, the measured data would allow the calculation of accurate empirical BER characteristics for Rayleigh fading environments.

\section{CONCLUSIONS}

Thanks to the modular design of the transmitter, the hardware communication system is suitable for MISO space-time coding techniques. It allows hardware reconfigurations by simply adding or removing RF modules from the control unit, as well as software modifications for implementing various forms of space-time coding. Further miniaturization of the control unit is also possible by implementing the central core and SRAM memories in a Field Programmable Gate Array (FPGA), making the control unit more cost-effective.

The measuring results in Figure 9 demonstrate the correct functionality of the developed hardware communications system, however with non-ideal performance due to phase noise. This proves that a good channel estimation on the receiver side is essential in order to correctly decode the received space-time coded symbols. To accomplish this, the developed hardware system still needs further optimization before it is used for measurements to empirically support the theoretical BER performance characteristics in [4] and [5].

In conclusion, with additional optimization, the developed hardware system is suitable for empirical characterization of space-time coding techniques. The system is low-cost, employs standard off-the-shelf components and is fully reconfigurable thanks to the modular design and the direct interfacing of embedded software to the hardware.

\section{REFERENCES}

[1] C.E. Shannon, A Mathematical Theory of Communication, The Bell System Technical Journal, vol. 27, pp. 379-423, 623-656, Jul. 1948

[2] J. Verhaevert, P. Van Torre, Realization and MIMO-link Measurements of a Transmit Module for Spatial Modulation, 9th European Conference on Antennas and Propagation (EuCAP2015), Lisbon, Portugal, 2015

[3] J. Verhaevert, P. Van Torre, A Low-cost and Modular Receiver for MIMO SDR, 10th European Conference on Antennas and Propagation (EuCAP2016), Davos, Switzerland, 2016

[4] M. Alamouti, A Simple Transmit Diversity Technique for Wireless Communications, IEEE Journal on Select Areas in Communications, vol. 16, No. 8, pp. 1451-1458, Oct. 1998.

[5] H. Jafarkhani, Space-Time coding: Theory and Practice, Cambridge Univ. Press, 2005, ISBN 978-0-521-84291-4

[6] J. Verhaevert, P. Van Torre, Design and Realization of a $2.45 \mathrm{GHz}$ Transmitter and Receiver as a Modular Unit for a MIMO SDR, Loughborough Antennas \& Propagation Conference (LAPC2015), Loughborough, United Kingdom, 2015

[7] MATLAB R2011, http://nl.mathworks.com/products/matlab/

[8] RTL2832U Mini DVB-T USB Dongle, http://www.dx.com/p/ rt12832u-r820t-mini-dvb-t-dab-fm-usb-digital-tv-dongle-black-170541 